Supporting Information

\title{
Raman spectroscopy-based 3D analysis of odontogenic differentiation of human dental pulp stem-cell spheroids
}

\author{
Huijung Kim ${ }^{a, 1}$, Yoojoong Han ${ }^{b, 1}$, Intan Rosalina Suhito ${ }^{a, 1}$, Yoon Choi ${ }^{a}$, Minkyeong Kwon ${ }^{a}$, \\ Hyungbin Son ${ }^{a, *}$, Hyung-Ryong Kim ${ }^{c,}{ }^{*}$,Tae-Hyung Kim ${ }^{a, d, *}$
}

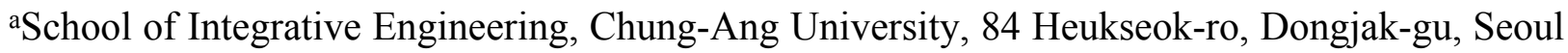
06974, Republic of Korea

${ }^{b}$ R\&D division, Nanobase, Inc., Seoul 08502, Republic of Korea

${ }^{\mathrm{c} C o l l e g e ~ o f ~ D e n t i s t r y, ~ D a n k o o k ~ U n i v e r s i t y, ~ C h e o n a n ~ 31116, ~ R e p u b l i c ~ o f ~ K o r e a ~}$

dntegrative Research Center for Two-Dimensional Functional Materials, Institute of Interdisciplinary Convergence Research, Chung-Ang University, Seoul 06974, Republic of Korea ${ }^{1}$ These authors contributed equally to this work

*To whom correspondence should be addressed

\section{Prof. Hyungbin Son}

Email: being@cau.ac.kr

\section{Prof. Hyung-Ryong Kim}

Email: hrkimdp@gmail.com

\section{Prof. Tae-Hyung Kim}

Email: thkim0512@cau.ac.kr

Website: https://bestlaboratory.wixsite.com/best 


\section{Table of Contents}

Table of Contents

\section{Supporting Figures}

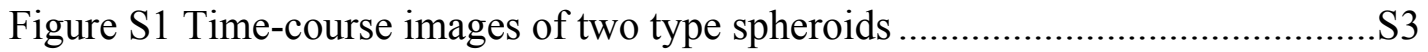

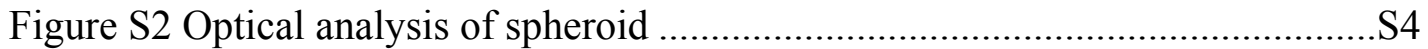

Figure S3 Optimization of Raman exposure time .................................................. 5

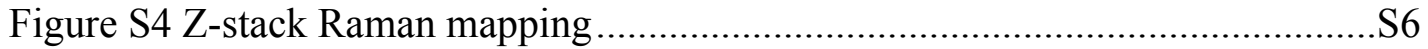

Figure S5 Raman mapping of High-density spheroid .........................................S7

Figure S6 Raman mapping of Low-density spheroid at point $1-7$.........................S8

Figure S7 Raman mapping of Low-density spheroid at point $8-13$.......................S9

Figure S8 PDMS molds used in Raman measurement ..........................................S10

\section{Supporting Tables}

Table S1 Primer sequences 


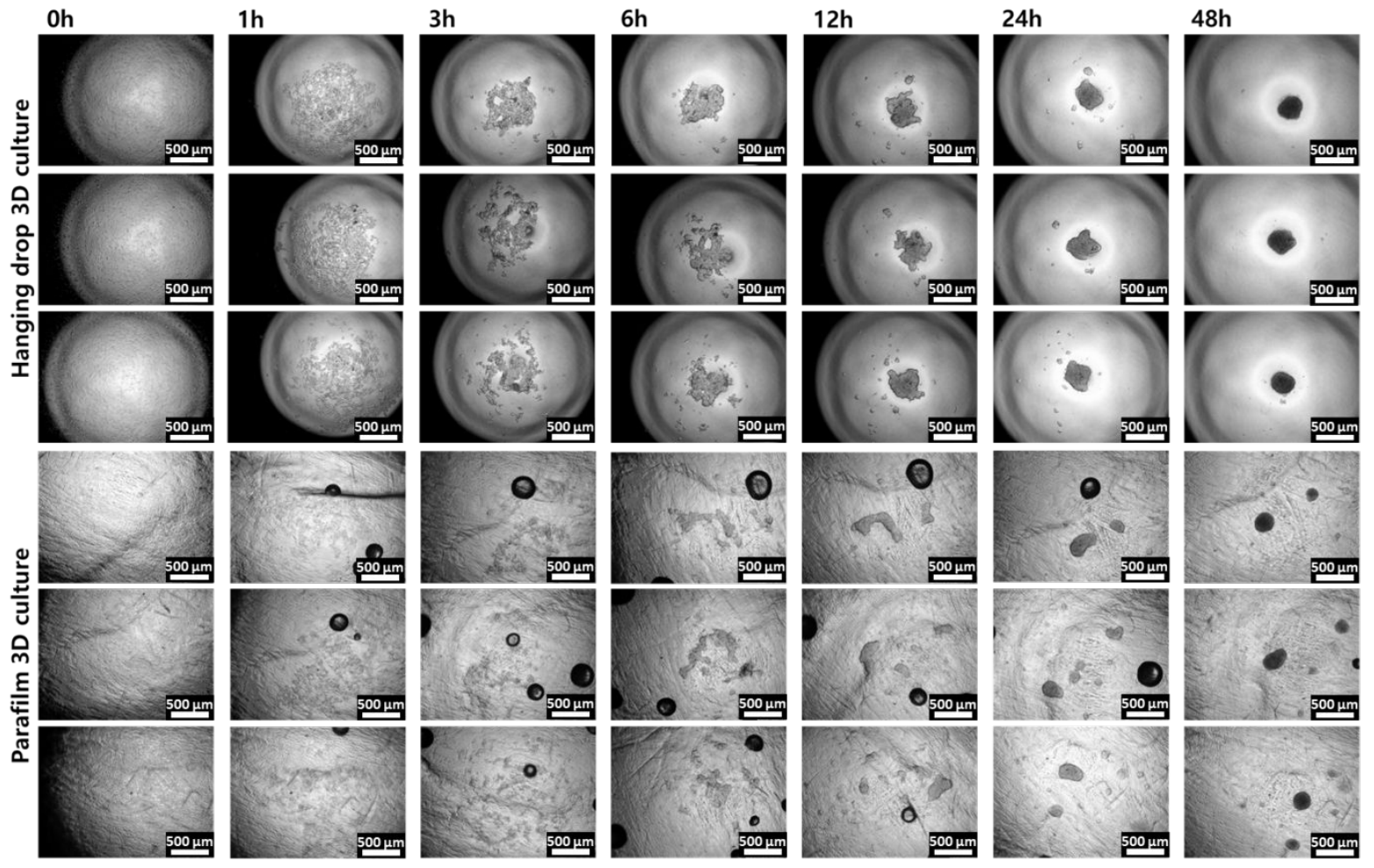

Figure S1. Time-course images of human dental pulp stem-cell (hDPSC) 3D spheroids generated using the hanging drop (HD) and molded parafilm-based (MP) methods. 
(a)

Spheroids of Hanging drop method

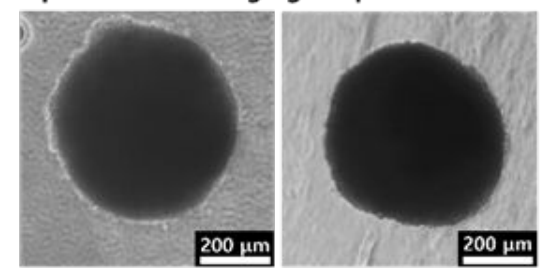

Spheroids of Parafilm method
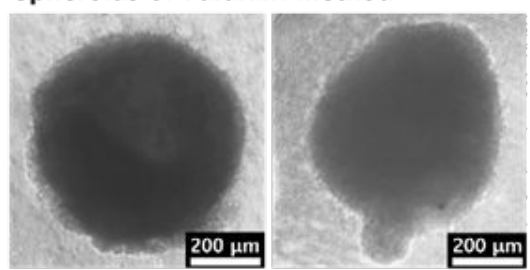

(c)

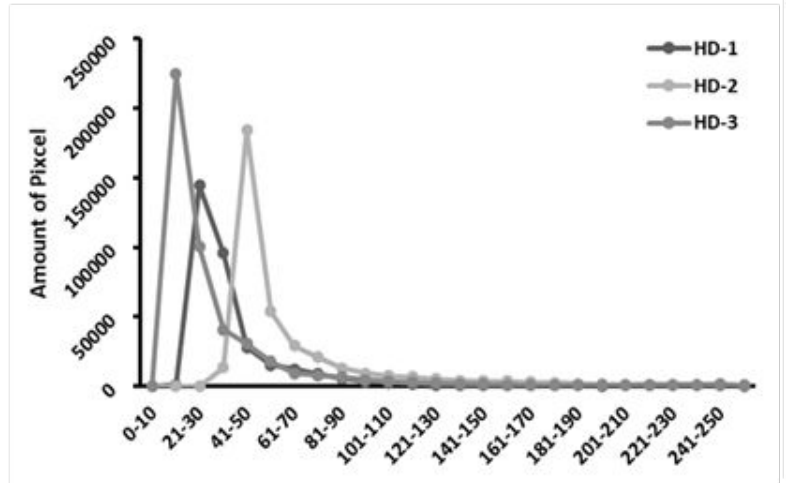

(b)
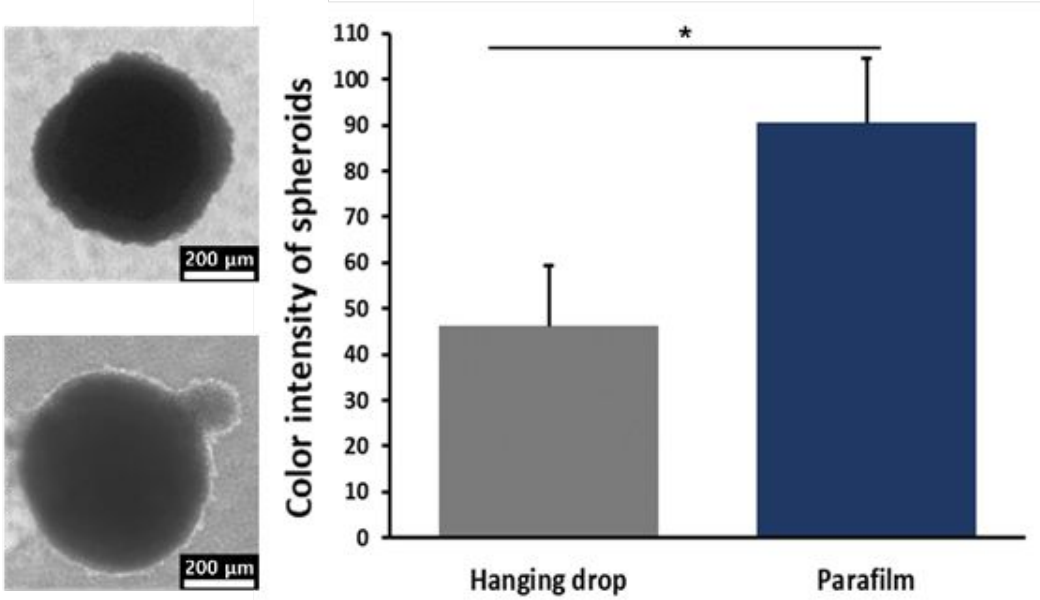

Figure S2. Optical images (a), color intensity (b), and intensity plot distribution (c) of HD- and MP-based spheroids $(n=3)$. 
(a)
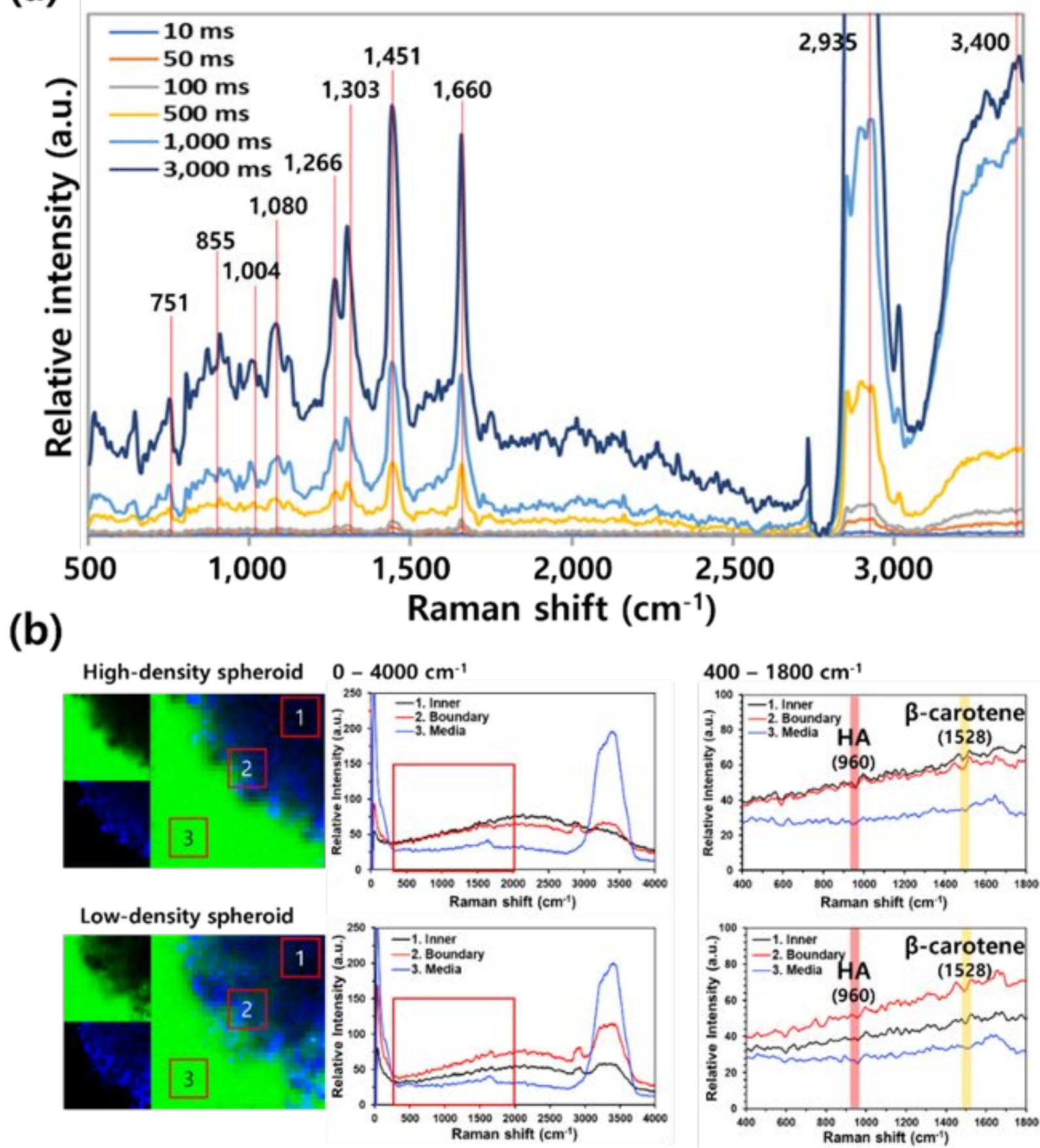

Figure S3. Optimization of Raman exposure time for the spheroid analysis (a), Raman mapping analysis of HD- and MP-based stem-cell spheroids, focusing on the spectral range of 400-1800 $\mathrm{cm}^{-1}$ (b). Raman mapping area: $100 \times 100 \mu \mathrm{m}$. 


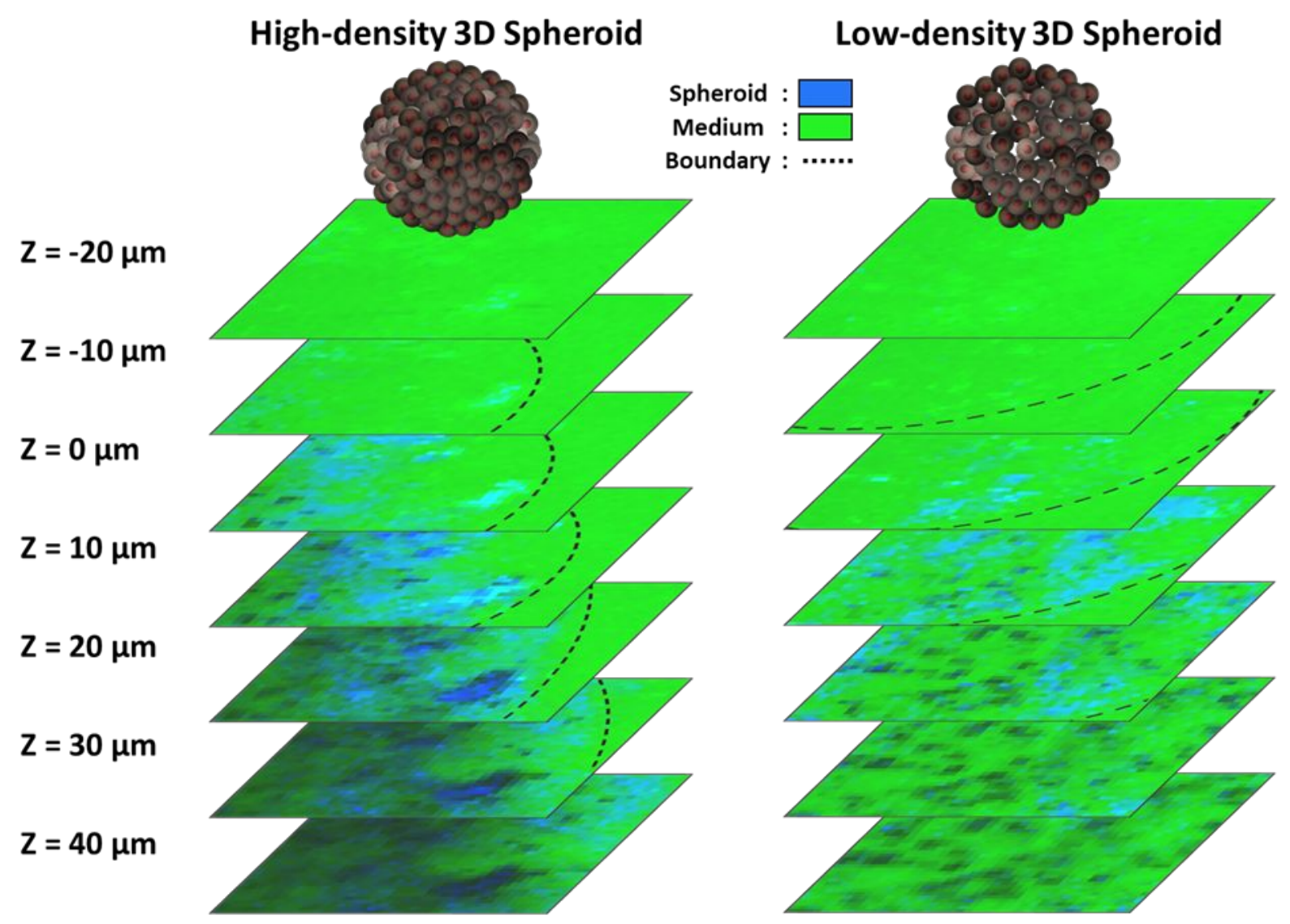

Figure S4. Raman mapping is based on the Z-stack position in hDPSC spheroids. Raman mapping analysis at the center of the spheroid at -20 to $40 \mu \mathrm{m}$. Corresponding Raman spectra data prior to the analysis are as follows: $\mathrm{H}_{2} \mathrm{O}$ peak at $3400 \mathrm{~cm}^{-1}$ and $\mathrm{CH}_{3}$ peak at $2935 \mathrm{~cm}^{-1}$. The area of Raman mapping was $80 \times 80 \mu \mathrm{m}$. 


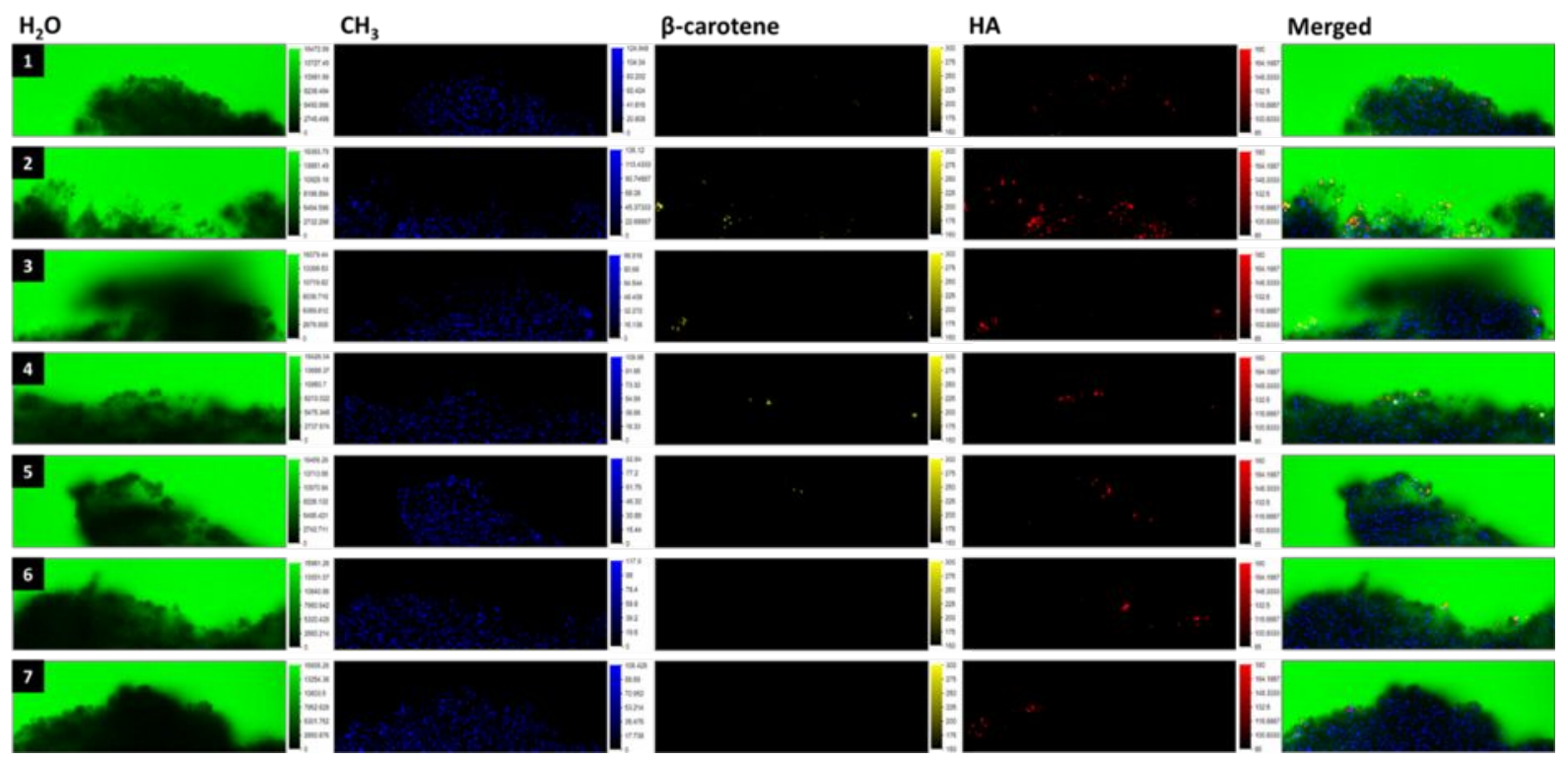

Figure S5. Raman mapping analysis at the edge regions at points 1-7 in high-density hDPSC spheroids. Corresponding Raman spectra data prior to the analysis are as follows: the $\mathrm{H}_{2} \mathrm{O}$ peak at $3400 \mathrm{~cm}^{-1}, \mathrm{CH}_{3}$ peak at $2935 \mathrm{~cm}^{-1}, \beta$-carotene peak at $1156 / 1528 \mathrm{~cm}^{-1}$, and the hydroxyapatite (HA, $\mathrm{PO}_{4}$ ) peak at $960 \mathrm{~cm}^{-1}$. The area of Raman mapping was $300 \times 60 \mu \mathrm{m}$. 


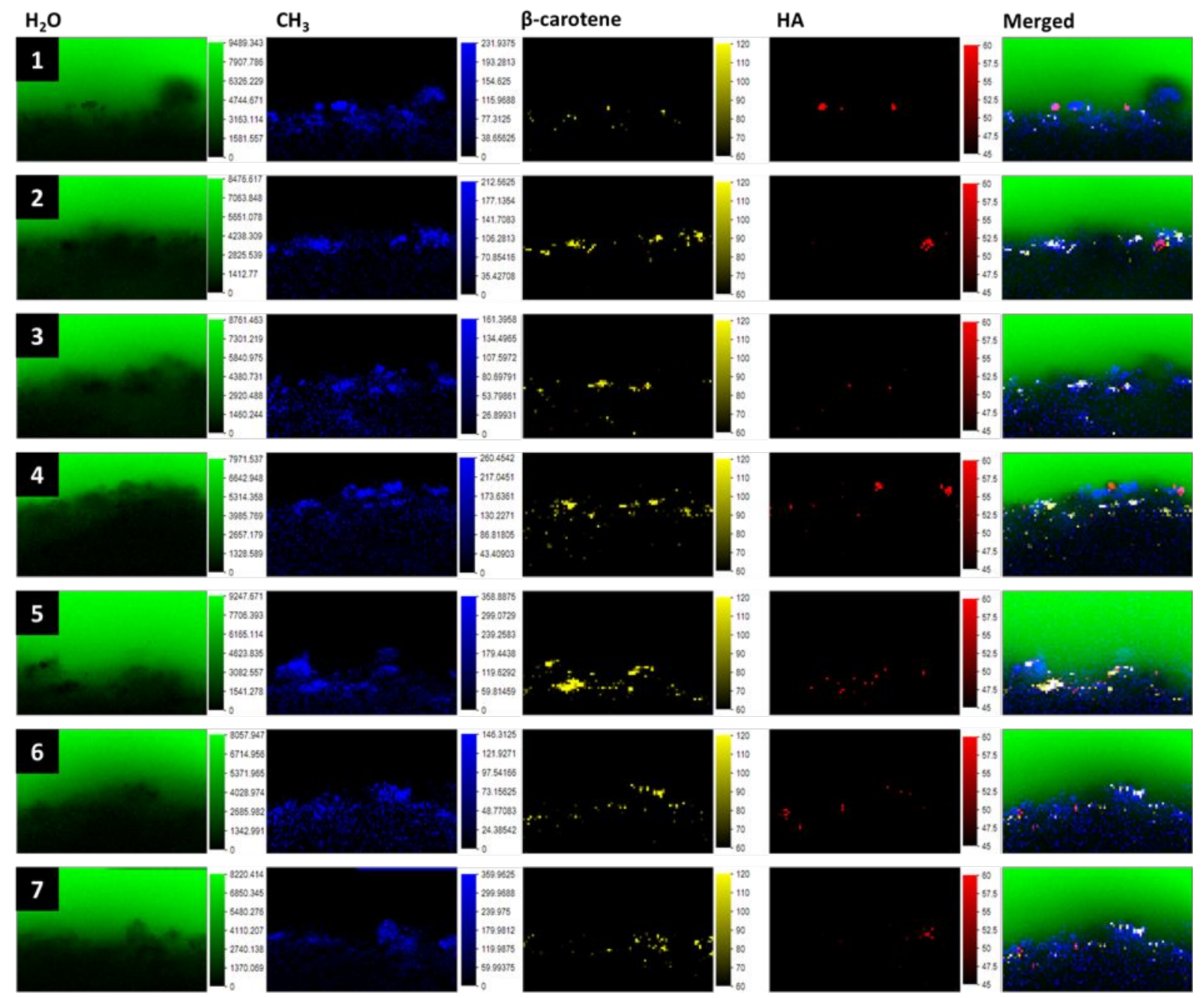

Figure S6. Raman mapping analysis at the edge at points 1-7 in low-density hDPSC spheroids. Corresponding Raman spectra data prior to the analysis are as follows: $\mathrm{H}_{2} \mathrm{O}$ peak at $3400 \mathrm{~cm}^{-1}$, $\mathrm{CH}_{3}$ peak at $2935 \mathrm{~cm}^{-1}, \beta$-carotene peak at $1156 / 1528 \mathrm{~cm}^{-1}$, and hydroxyapatite $\left(\mathrm{HA}, \mathrm{PO}_{4}\right)$ peak at $960 \mathrm{~cm}^{-1}$. The area of Raman mapping was $90 \times 60 \mu \mathrm{m}$. 


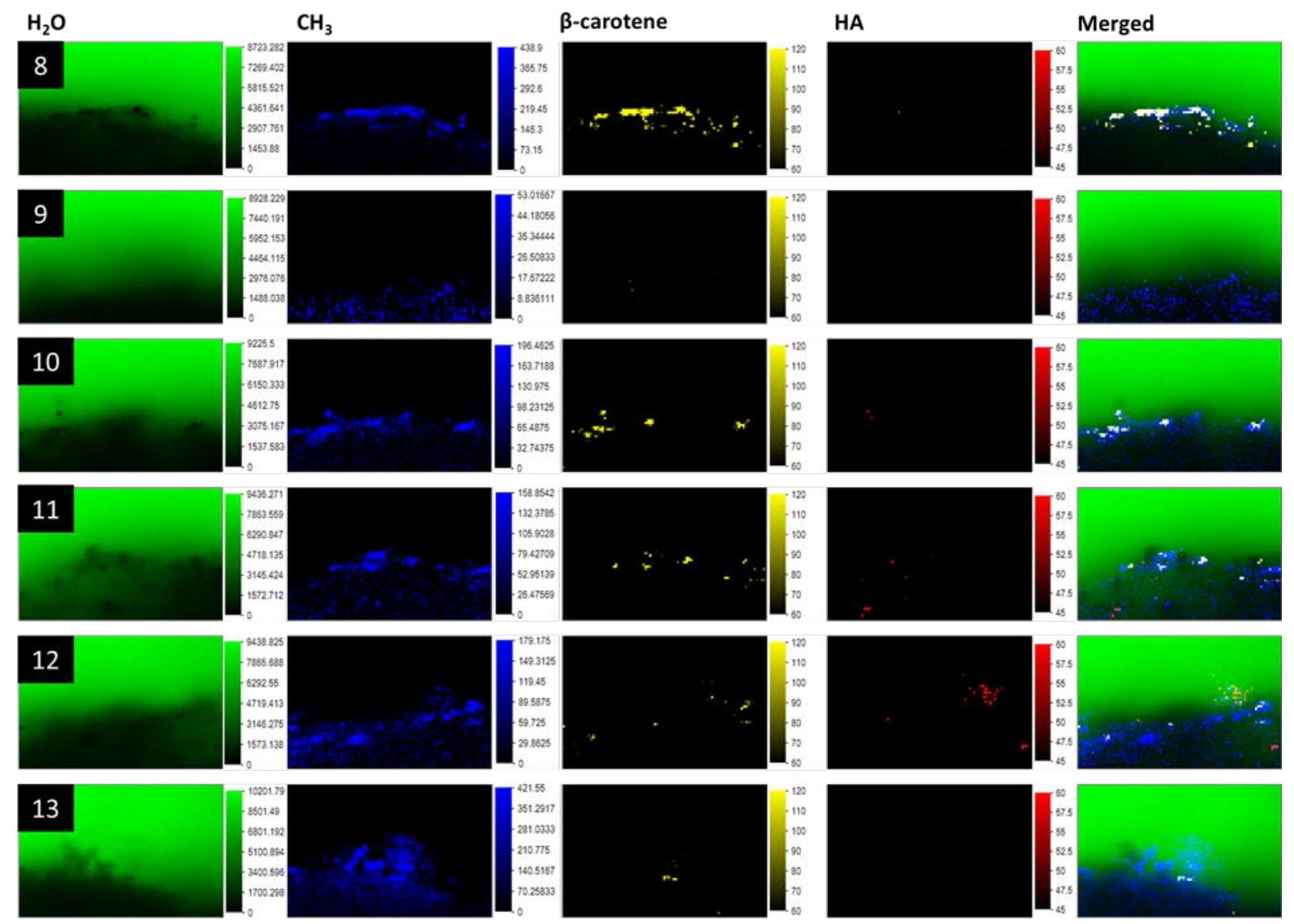

Figure S7. Raman mapping analysis at the edge at points 8-13 in low-density hDPSC spheroids.

Corresponding Raman spectra data prior to the analysis are as follows: the $\mathrm{H}_{2} \mathrm{O}$ peak at $3400 \mathrm{~cm}^{-1}$, $\mathrm{CH}_{3}$ peak at $2935 \mathrm{~cm}^{-1}, \beta$-carotene peak at $1156 / 1528 \mathrm{~cm}^{-1}$, and hydroxyapatite $\left(\mathrm{HA}, \mathrm{PO}_{4}\right)$ peak at $960 \mathrm{~cm}^{-1}$. The area of Raman mapping was $90 \times 60 \mu \mathrm{m}$. 

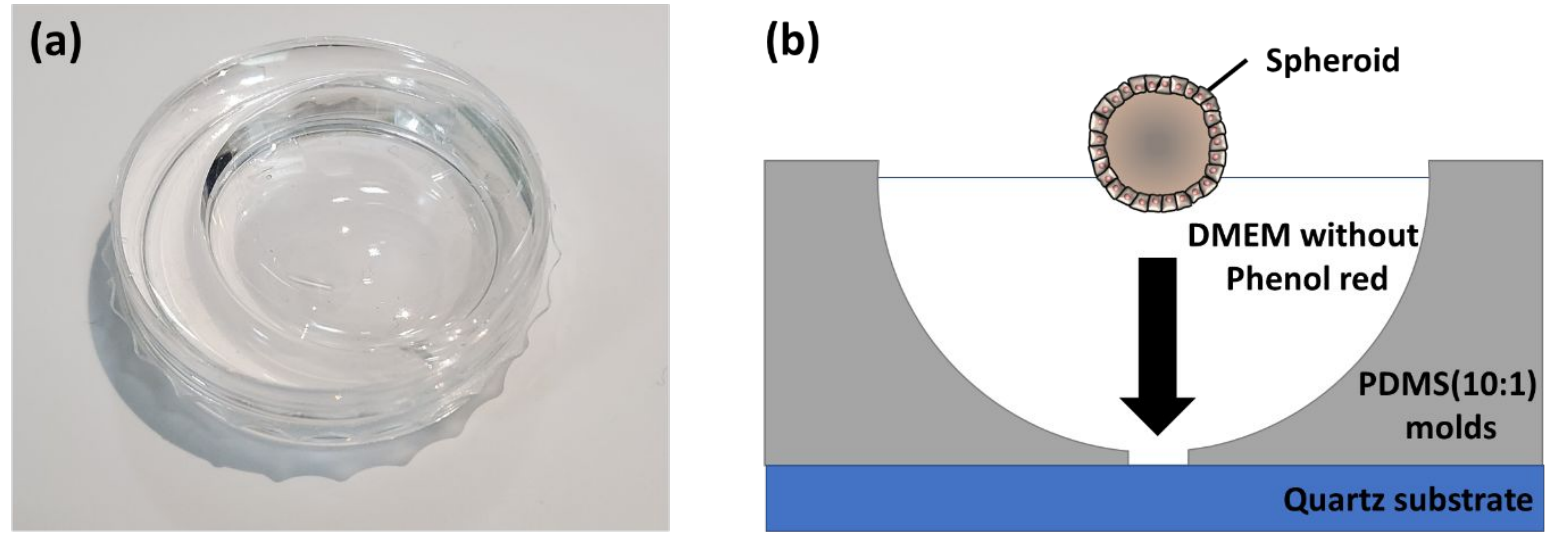

Figure S8. Polydimethylsiloxane (PDMS) (10:1) mold images (a) and spheroid analysis diagram (b) used for Raman measurement. 


\begin{tabular}{lll}
\hline Target gene & Primer sequence $\left(5^{\prime}-3^{\prime}\right)$ \\
\hline \multirow{2}{*}{ Runx2 } & Forward & GGCCCACAAATCTCAGATCGTT \\
& Reverse & CACTGGCGCTGCAACAAGAC \\
ALP & Forward & AACACCACCCAGGGGAAC \\
& Reverse & GGTCACAATGCCCACAGATT \\
OCN & Forward & GGCGCTACCTGTATCAATGG \\
& Reverse & TCAGCCAACTCGTCACAGTC \\
DSPP & Forward & TTCGATGGGAGTCCTAGTG \\
& Reverse & TGAGCTTCTGGGTGTCCTCT \\
& & CCTGAGGATGAGAACAGCTCC \\
DMP1 & Forward & A \\
& Reverse & GATCTGCTGTCTTCTTGAGAGC \\
& & FTGGCGCTGAGTACGTCG \\
& Revward & TTGACAAAGTGGTCGTTG
\end{tabular}

$\overline{\text { Table S1. Primer sequences used for the amplification of housekeeping gene and differentiation }}$ marker genes in RT-qPCR. 